\title{
Stacking Fault Energy of Si Nanocrystals Embedded in $\mathrm{SiO}_{2}$
}

\author{
Y. Q. Wang, ${ }^{1}$ W. S. Liang, ${ }^{1}$ and G. G. Ross ${ }^{2}$ \\ ${ }^{1}$ The Cultivation Base for State Key Laboratory, Qingdao University, No. 308, Ningxia Road, Qingdao 266071, China \\ ${ }^{2}$ Group of Ion Implantation, INRS-EMT, 1650 Boulevard Lionel-Boulet, Varennes, QC, Canada J3X 1S2
}

Correspondence should be addressed to Y. Q. Wang, yqwang@qdu.edu.cn

Received 16 February 2011; Accepted 4 April 2011

Academic Editors: M. Cazzanelli, G. A. Kachurin, and D. Tsoukalas

Copyright () 2011 Y. Q. Wang et al. This is an open access article distributed under the Creative Commons Attribution License, which permits unrestricted use, distribution, and reproduction in any medium, provided the original work is properly cited.

\begin{abstract}
Si nanocrystals ( $\mathrm{Si} \mathrm{nc}$ ) were produced by the implantation of $\mathrm{Si}^{+}$into a $\mathrm{SiO}_{2}$ film on (100) $\mathrm{Si}$, followed by high-temperature annealing. High-resolution transmission electron microscopy (HRTEM) observation has shown that a perfect dislocation (Burgers vector $\mathbf{b}=(1 / 2)\langle 110\rangle)$ can dissociate into two Shockley partials (Burgers vector $\mathbf{b}=(1 / 6)\langle 112\rangle$ ) bounding a strip of stacking faults (SFs). The width of the SFs has been determined from the HRTEM image, and the stacking fault energy for Si nc has been calculated. The stacking fault energy for Si nc is compared with that for bulk Si, and the formation probability of defects in Si nc is also discussed. The results will shed a light on the dissociation of dislocations in nanoparticles.
\end{abstract}

\section{Introduction}

Si nanocrystals ( $\mathrm{Si} \mathrm{nc}$ ) embedded in a $\mathrm{SiO}_{2}$ matrix have attracted much attention as a promising candidate for optoelectronics. It has been agreed by most researchers that the defect center at the $\mathrm{Si} \mathrm{nc} / \mathrm{SiO}_{2}$ interface plays an important role in the light emission $[1,2]$. In order to fully understand light emission mechanism, a comprehensive investigation of the microstructure of the Si nc and their surrounding matrix, especially the defects inside the Si nc or at the interface of $\mathrm{Sinc} / \mathrm{SiO}_{2}$, is necessary.

When analyzing the reasons for the formation of defects in the nanocrystals, the following two factors are usually considered [3]: the size effect and the energy of formation of stacking faults (SFs). The size effect on the formation of defects has been discussed in our previous paper [4]. It has been pointed out that the probability of defects being formed drops sharply when the Si nc are smaller than $5 \mathrm{~nm}$ [4]. The probability of defects, for example, SFs, being formed also depends on their energy of formation. However, the influence of stacking fault energy on the formation of defects in Si nc has not been reported yet.

\section{Experimental}

A $1 \mu \mathrm{m}$ thick film of amorphous $\mathrm{SiO}_{2}$ was obtained from universitywafer.com. It was produced by thermal oxidation of (100) Si substrate at high temperature $\left(\sim 1100^{\circ} \mathrm{C}\right.$ under oxygen flow). The amorphous $\mathrm{SiO}_{2}$ film was implanted at room temperature with $100 \mathrm{keV} \mathrm{Si}^{+}$(implantation dose of $3 \times 10^{17} \mathrm{~cm}^{-2}$ ), followed by high-temperature annealing at $1100^{\circ} \mathrm{C}$ for $1 \mathrm{~h}$ under an atmosphere of nitrogen $\left(\mathrm{N}_{2}\right)$. After the implantation, hydrogen passivation was carried out at $500^{\circ} \mathrm{C}$ for $1 \mathrm{~h}$ in a forming gas of $\mathrm{H}_{2} \quad(5 \%)$ and $\mathrm{N}_{2}(95 \%)$. The specimens for transmission electron microscopy examination were prepared in a cross-sectional orientation ([011] zone axis for the Si substrate) using conventional techniques of mechanical polishing, dimpling, and ion thinning. The ion thinning was performed using a Gatan model 691 PIPS. Dark field (DF) examination was carried out on a Philips CM30 microscope operating at $300 \mathrm{kV}$. High-resolution transmission electron microscopy (HRTEM) observations were performed using a JEOL JEM $2100 \mathrm{~F}$ transmission electron microscope operating at $200 \mathrm{kV}$.

\section{Results and Discussion}

Figure 1(a) shows a typical cross-sectional DF image of this specimen [5]. From Figure 1(a), it can be seen that the Si nc layer extends from $50 \pm 5 \mathrm{~nm}$ to a maximum depth of $290 \pm$ $10 \mathrm{~nm}$, and the size ranges from 2 to $22 \mathrm{~nm}$ in diameter. The Si nc can be divided into three regions, RI, RII, and RIII, according to their sizes. The Si nc in the middle region of 


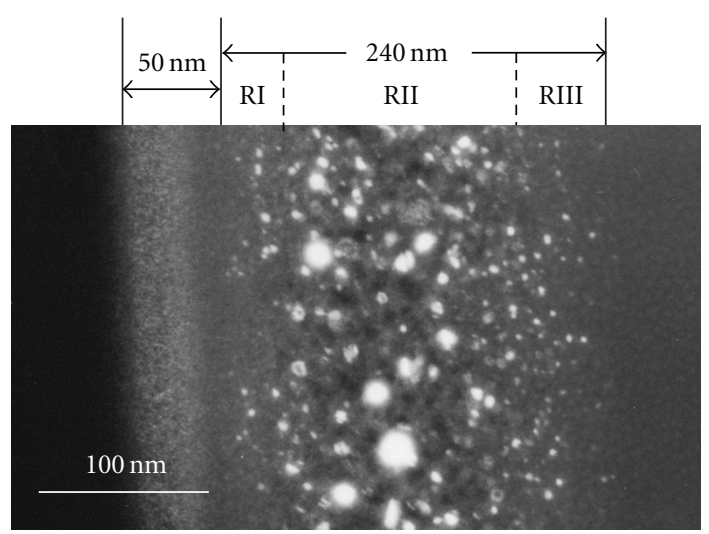

(a)

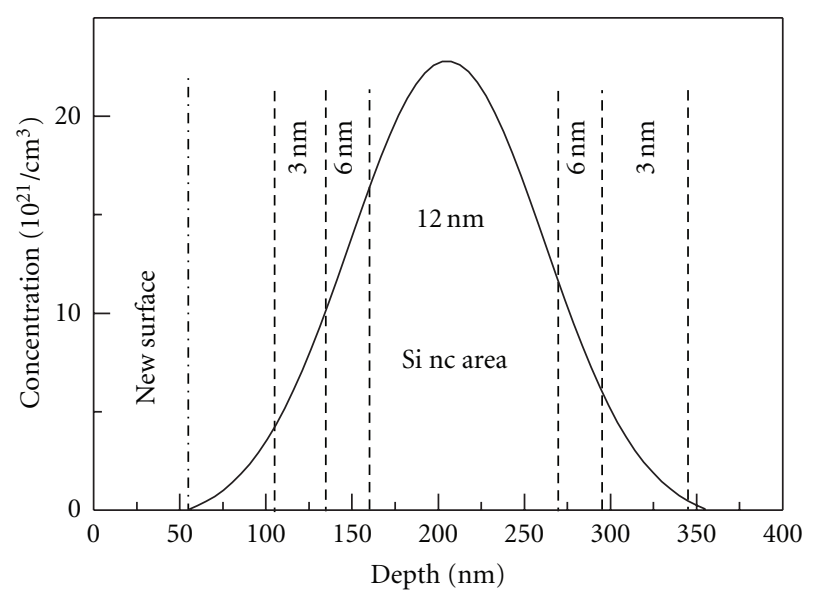

(b)

FIGURE 1: (a) Typical cross-sectional DF image of the specimen; (b) simulated depth profile of $\mathrm{Si}$ implanted $(100 \mathrm{keV})$ into $\mathrm{SiO}_{2}$ with a fluence of $3 \times 10^{17} \mathrm{~cm}^{-2}$.

the implanted layer are larger than those near the free surface or the bottom of the implanted layer, which is in agreement with a Gaussian-shaped excess Si concentration depth profile in Figure 1(b). Figure 1(b) is the depth profile of Si nc obtained by means of SRIM code [6], and the sputtering effect has been considered. In Figure 1(b), the average sizes of the $S i \mathrm{nc}$ are divided into three major groups: 3, 6, and $12 \mathrm{~nm}$. From Figure 1, it can be seen that the simulated depth profile is consistent with that obtained from TEM observation.

Extensive HRTEM observations of the Si nc show that a perfect dislocation can dissociate into two Shockley partials (Burgers vector $\mathbf{b}=(1 / 6)\langle 112\rangle)$ bounding a strip of SFs. Figure 2(a) shows a typical HRTEM image of a Si nanocrystal with an extended dislocation [6], where the particle is oriented along the [011] direction. The size of the Si nanocrystal is around $15 \mathrm{~nm}$. It can be seen from Figure 2(a) that two Shockley partial dislocations are connected by a strip of SFs. The two Shockley partials are indicated by two arrows and labeled as $30^{\circ}$ and $90^{\circ}$, and the dislocation line directions are along [011]. The SFs have a characteristic of an intrinsic stacking fault with a displacement of $\mathbf{R}=$ $-1 / 3\langle 111\rangle[7]$. In order to demonstrate the configuration

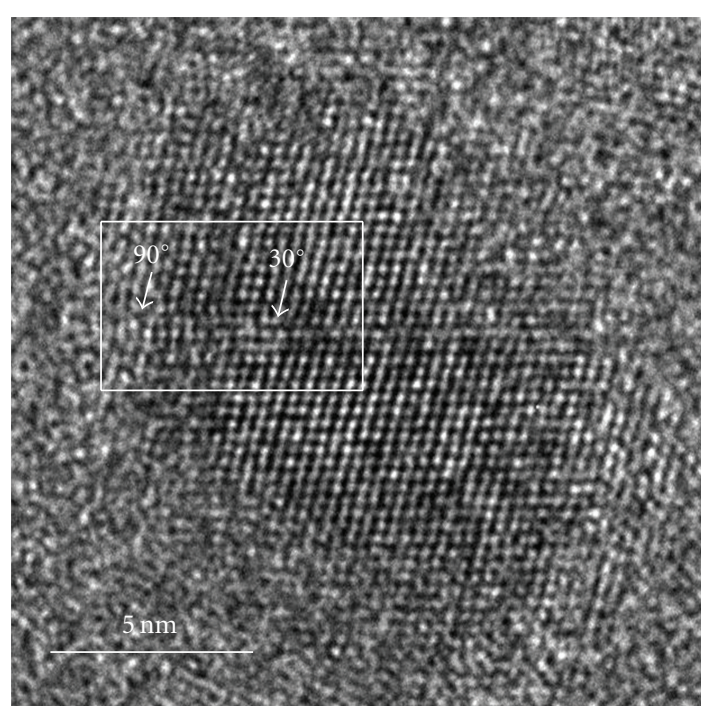

(a)

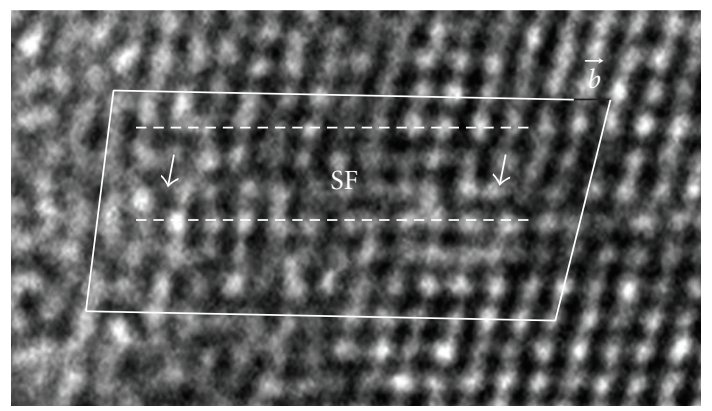

(b)

FIgure 2: (a) [011] zone-axis HRTEM image of a typical Si nanoparticle with an extended dislocation; (b) close-up of the extended dislocation showing two Shockley partials bounding a strip of SFs.

more clearly, the enlarged HRTEM image of the rectangleenclosed region in Figure 2(a) is shown in Figure 2(b). The Burgers circuit enclosing the two Shockley partials (indicated by two arrows) is drawn in Figure 2(b). From the Burgers circuit, it can be clearly seen that there is a gap between the starting and ending point, which is indicated by an arrow. The total Burgers vector is determined to be $1 / 2\langle 110\rangle$. In addition, the SFs region is enclosed by the dashed lines in Figure 2(b). The width of SFs is measured to be $2.97 \pm$ $0.33 \mathrm{~nm}$ from Figure 2(b).

There are mainly two factors exerting influences on the width of SFs: the interactions between two partial dislocations and the stacking fault energy. On one hand, dislocations lines repel each other with a force $f$ per unit length; on the other hand, stacking fault energy per unit area, $\gamma$, forces the dislocation lines to move closer. At equilibrium, $f=\gamma$, the SF width $\left(d_{0}\right)$ can be described as $[8,9]$

$$
d_{0}=G \mathbf{b} 1 \cdot \frac{\mathbf{b} 2}{\gamma},
$$

where $G$ is the shear modulus and $\mathbf{b}_{1}$ and $\mathbf{b}_{2}$ are the Burgers vectors for two partial dislocations. 
In deformed bulk $\mathrm{Si}$, the $\mathrm{SFs}$ and the dissociation of a perfect dislocation (Burgers vector $\mathbf{b}=(1 / 2)\langle 110\rangle$ ) into two Shockley partial dislocations (Burgers vector $\mathbf{b}=$ $(1 / 6)\langle 112\rangle)$ were observed using the weak-beam darkfield technique [10,11] and HRTEM [12]. Experimental observations indicated that the size of the stacking faults range from 30 to $50 \AA$ [11-13], and the value for the intrinsic stacking faults energy is calculated to be $55 \pm 7 \mathrm{~mJ} / \mathrm{m}^{2}$ $[10,11,14]$ or $69 \mathrm{~mJ} / \mathrm{m}^{2}[15,16]$. However, the stacking fault energy for Si nc has not been determined yet.

The equilibrium width for SFs in the Si nc $\left(\mathrm{d}_{\mathrm{Sinc}}\right)$ has been measured to be about $2.97 \pm 0.33 \mathrm{~nm}$, so the stacking fault energy can be calculated using (1). The value for $G$ of $6.36 \times 10^{10} \mathrm{~N} / \mathrm{m}^{2}$ is used [17], which is the effective value for defects on $\{111\}$ planes. The SF energy for the Si nc $\left(\gamma_{\mathrm{Si} \mathrm{nc}}\right)$ is estimated to be $84 \pm 9 \mathrm{~mJ} / \mathrm{m}^{2}$, which is much higher than that for bulk Si reported in the literature $[10,11,14-$ 17]. Therefore, we can deduce that the probability for the formation of SFs in Si nc is less than that in bulk Si. Usually in the nanoparticles, dislocations can slip through the particle and no evident boundary can be observed between the perfect and faulted lattices [7]. Here we observed the dissociation of a perfect dislocation into two partial dislocations. The partial dislocations can be pinned at the $\mathrm{Si}$ $\mathrm{nc} / \mathrm{SiO}_{2}$ interface where some impurities are located [18].

\section{Conclusions}

In conclusion, extended dislocations have been observed in the Si nanocrystals using HRTEM. The width of the SFS is measured to be $2.97 \pm 0.33 \mathrm{~nm}$, and the stacking fault energy is calculated to be $84 \pm 9 \mathrm{~mJ} / \mathrm{m}^{2}$. The probability for the formation of SFs in Si nc is less than in bulk Si.

\section{Acknowledgments}

This paper was financially supported by the Scientific Research Award Foundation for Outstanding Young and Middle-Aged Scientists in Shandong Province (Grant no. BS2009CL005), the Natural Science Foundation for Excellent Young Scientists in Shandong Province (Grant no. JQ201002), and the Scientific Research Starting Foundation for Introduced Talents at Qingdao University (Grant no. 06300701). One author (Y. Q. Wang) would also like to thank the financial support from Taishan Outstanding Overseas Scholar Program of Shandong Province.

\section{References}

[1] L. N. Dinh, L. L. Chase, M. Balooch, W. J. Siekhaus, and F. Wooten, "Optical properties of passivated Si nanocrystals and $\mathrm{SiO}_{2}$ nanostructures," Physical Review B, vol. 54, no. 7, pp. 5029-5037, 1996.

[2] T. Shimizu-Iwayama, N. Kurumado, D. E. Hole, and P. D. Townsend, "Optical properties of silicon nanoclusters fabricated by ion implantation," Journal of Applied Physics, vol. 83, no. 11, pp. 6018-6022, 1998.

[3] G. E. Abrosimova and A. S. Aronin, "The fine structure of FCC nanocrystals in Al- and Ni-based alloys," Physics of the Solid State, vol. 44, no. 6, pp. 1003-1007, 2002.
[4] Y. Q. Wang, R. Smirani, and G. G. Ross, "Nanotwinning in silicon nanocrystals produced by ion implantation," Nano Letters, vol. 4, no. 10, pp. 2041-2045, 2004.

[5] Y. Q. Wang, R. Smirani, and G. G. Ross, "The effect of implantation dose on the microstructure of silicon nanocrystals in $\mathrm{SiO}_{2}$," Nanotechnology, vol. 15, no. 11, pp. 1554-1560, 2004.

[6] Y. Q. Wang, T. Li, W. S. Liang, X. F. Duan, and G. G. Ross, "Dislocations in Si nanocrystals embedded in $\mathrm{SiO}_{2}$," Nanotechnology, vol. 20, no. 31, Article ID 315704, pp. 1-4, 2009.

[7] Y. Q. Wang, R. Smirani, and G. G. Ross, "Stacking faults in Si nanocrystals," Applied Physics Letters, vol. 86, no. 22, Article ID 221920, 3 pages, 2005.

[8] J. P. Hirth and J. Lothe, Theory of Dislocations, Wiley, New York, NY, USA, 1982.

[9] I. Kovács and L. Zsoldos, Dislocations and Plastic Deformation, Pergamon Press, Oxford, UK, 1973.

[10] F. Häussermann and H. Schaumburg, "Extended dislocations in germanium," Philosophical Magazine, vol. 27, no. 3, pp. 745-751, 1973.

[11] I. L. F. Ray and D. J. H. Cockayne, "The dissociation of dislocations in silicon," Proceedings of the Royal Society A, vol. 325, pp. 543-554, 1971.

[12] A. Olsen and J. C. H. Spence, "Distinguishing dissociated glide and shuffle set dislocations by high resolution electron microscopy," Philosophical Magazine A, vol. 43, no. 4, pp. 945$965,1981$.

[13] J. L. Demenet, P. Grosbras, H. Garem, and J. C. Desoyer, "Dislocation dissociation widths in silicon at low temperature under controlled high-stress orientations," Philosophical Magazine A, vol. 59, no. 3, pp. 501-518, 1989.

[14] S. Takeuchi and K. Suzuki, "Stacking fault energies of tetrahedrally coordinated crystals," Physica Status Solidi A, vol. 171, no. 1, pp. 99-103, 1999.

[15] A. George and J. Rabier, "Dislocations and plasticity in semiconductors. I. Dislocation structures and dynamics," Revue de Physique Appliquée, vol. 22, no. 9, pp. 941-966, 1987.

[16] B. Joós, Q. Ren, and M. S. Duesbery, "Peierls-Nabarro model of dislocations in silicon with generalized stacking-fault restoring forces," Physical Review B, vol. 50, no. 9, pp. 58905898, 1994.

[17] E. Aerts, P. Delavignette, R. Siems, and S. Amelinckx, "Stacking fault energy in silicon," Journal of Applied Physics, vol. 33, no. 10, pp. 3078-3080, 1962.

[18] J. Y. Cheng, D. J. Eaglesham, D. C. Jacobson, P. A. Stolk, J. L. Benton, and J. M. Poate, "Formation of extended defects in silicon by high energy implantation of B and P," Journal of Applied Physics, vol. 80, no. 4, pp. 2105-2112, 1996. 

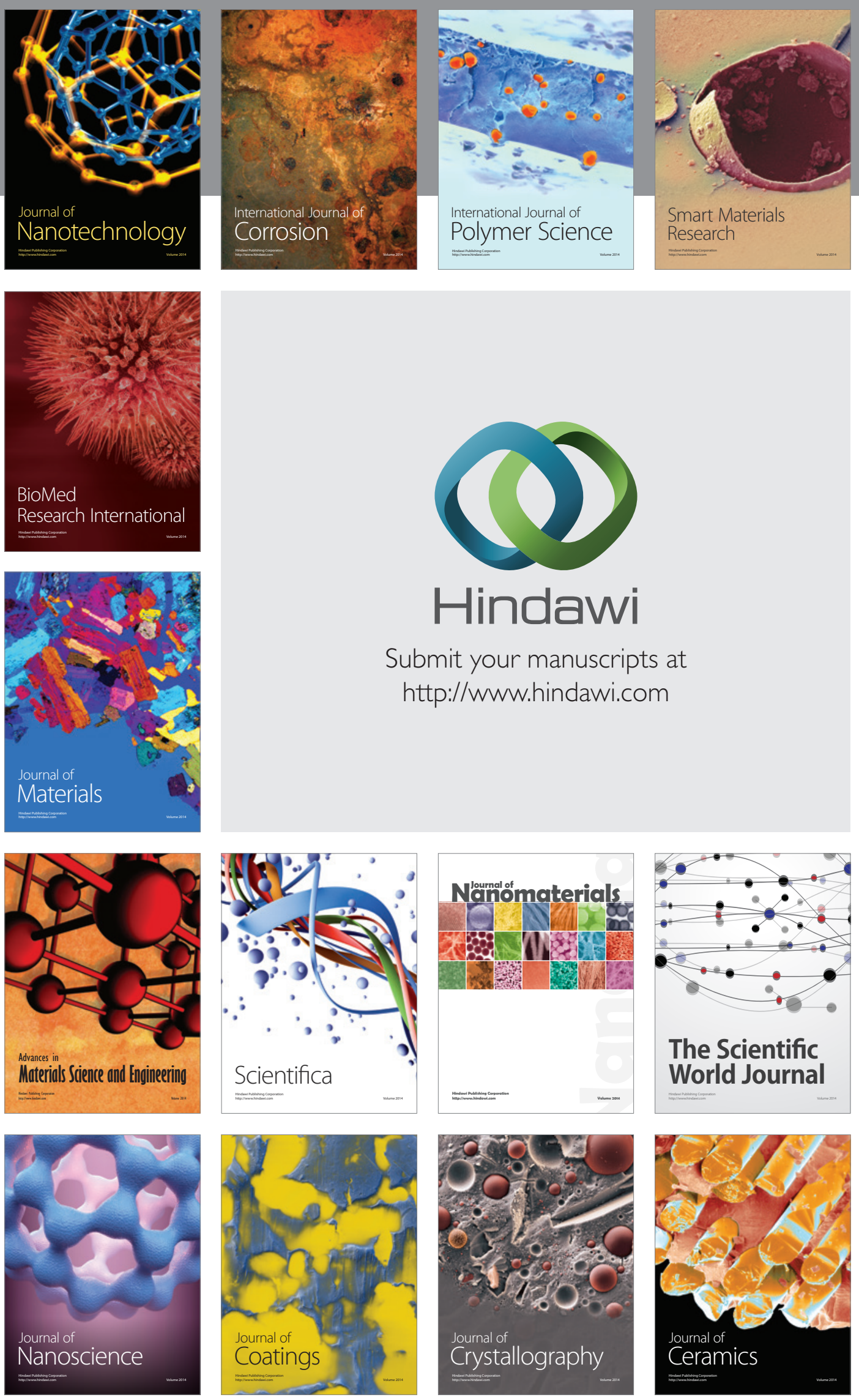

The Scientific World Journal

Submit your manuscripts at

http://www.hindawi.com

\section{World Journal}

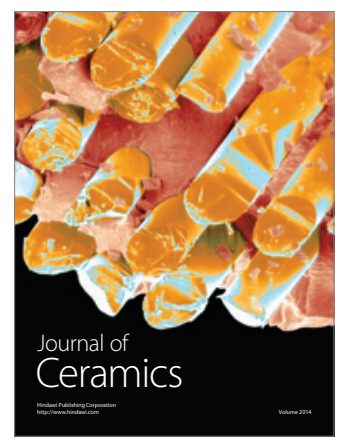

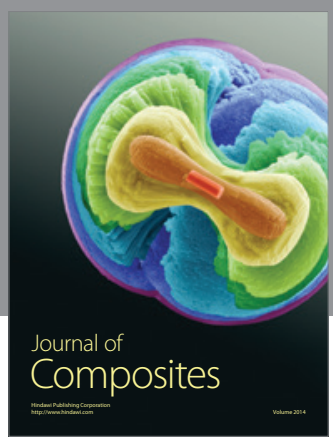
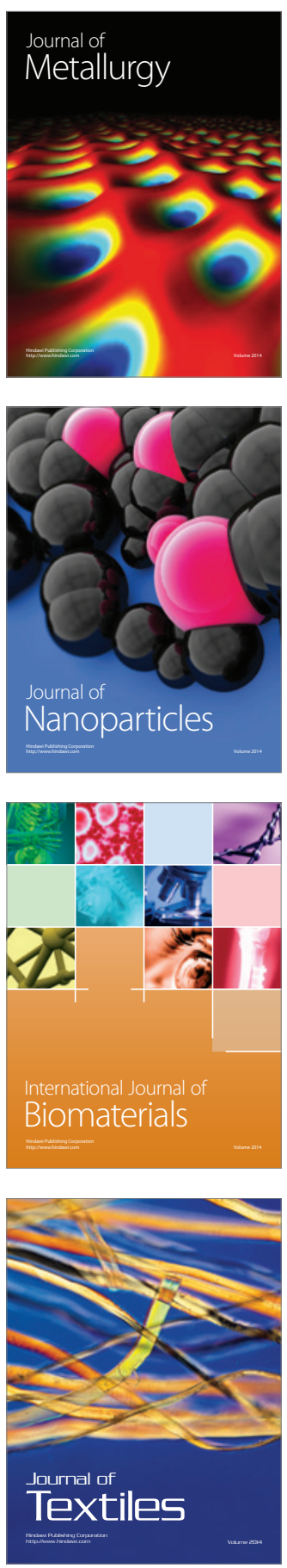